\section{GP60 CLINICAL UTILITY OF ELEVATED VITAMIN B12 LEVELS WITH A PROGRESSIVELY ABNORMAL BLOOD FILM}

${ }^{1}$ David Foley*, ${ }^{1}$ Patricia Fitzsimons, ${ }^{2}$ Andrea Malone, ${ }^{1}$ Ingrid Borovickova, ${ }^{1,2}$ Melanie Cotter. ${ }^{1}$ Childrens University Hospital Temple Street, Dublin, Ireland; ${ }^{2}$ Our Ladys's Children Hospital Crumlin, Dublin, Ireland

\subsection{6/archdischild-2019-epa.126}

Introduction Cobalamin plays an essential role in haematopoiesis, cell metabolism, production of DNA and neuronal function. High serum concentration of cobalamin is a common but underestimated finding. The aetiology of hypercobalaminaemia includes excess vitamin B12 intake, solid neoplasms, haematological malignancies, liver disease, renal failure and autoimmune diseases. Paradoxically, hypercobalaminaemia can be associated with a functional B12 deficiency. A finding of high serum cobalamin should prompt an early and in-depth search for these entities to ensure a favourable prognosis.

Case presentation We present a case of a thirteen-year old girl who presented with non-specific symptoms and an incidental finding of a raised serum cobalamin $(1400 \mathrm{pg} / \mathrm{mL})$ with initially only minor full blood count (FBC) abnormalities. Upon her assessment in the haematology clinic in OLCHC, repeat FBC results showed progressive anaemia with macrocytosis, a falling platelet count and serum cobalamin concentration of $>6000 \mathrm{pg} / \mathrm{mL}$. On repeat the cobalamin had increased to $10926 \mathrm{pg} / \mathrm{mL}$. Urinary methylmalonic acid, plasma total homocysteine, liver and renal function were unremarkable, and she was not taking supplements or oestrogens. A bone marrow aspirate showed findings consistent with early diagnosis of Acute Myeloid Leukaemia (AML).

Conclusion This case highlights the importance of hypercobalaminaemia which should be followed by the search for the cause of this finding as early diagnosis can be an important prognostic factor. A possible malignant blood disorder should be considered when serum cobalamin concentrations are above the reference range and where increases due to supplements, inflammatory, renal or liver disease have been excluded. This underscores the importance of laboratories offering numerical values (rather than reporting high results as greater than a cut-off value) for elevated vitamin B12 levels in cases with minor but progressive FBC changes. An increase in the level of B12 may indicate the need for prompt referral to a haematologist for assessment and monitoring.

\section{GP61 SWOLLEN EYES WITH AN UNPLEASANT SURPRISE - A CASE REPORT OF A STEROID RESISTANT NEPHROTIC SYNDROME}

Anna Stanzelova*, Anna Krepelova, Michaela Nemcikova, Jakub Zieg. University Hospital Motol, Prague, Czech Republic

\subsection{6/archdischild-2019-epa.127}

With a reported incidence of 7:100 000, nephrotic syndrome (NS) is one of the more common nephrology disorders that can present to and is often treated by the general paediatrician in a primary and secondary level hospital. Standard treatment consists of steroids. Steroid resistant NS (SRNS) is an indication for renal biopsy.

We present a case of a 5-year-old girl, who presented to a regional hospital at the age of 2.5 years, with periorbital oedema and abdominal swelling. Her medical history was significant for premature delivery, history of Intrauterine Growth Restriction (IUGR) and postnatal failure to thrive, persistent leucopoenia and mild developmental delay. Upon presentation she had marked proteinuria, serum hypoalbuminemia and hyperlipidaemia. A diagnosis of NS was made but she failed to respond to standard steroid treatment. She was transferred to a tertiary hospital on day 26 of admission with a suspicion of thrombophlebitis of her left lower limb. There, she was noted to have low set ears, areas of hyperpigmentation on her trunk, periorbital and limb oedema, ascites and abnormal posture. Pelvic X-ray showed bilateral hip dysplasia. Eye exam revealed retinal degeneration and blood investigations noted hypogammaglubulinemia, low CD4 and CD8 lymphocytes and high TSH. In view of her clinical picture, steroid resistant NS and leucopoenia, Shimke Immuno-Osseous dysplasia (SIOD) was suspected. SIOD is a rare autosomal recessive disease consisting of renal failure, skeletal dysplasia and T-cell deficiency. This was subsequently confirmed by genetic testing which showed 2 pathogenic mutations within the SMARCAL 1 gene. Overtime she progressed to end stage renal disease, and initiated peritoneal dialysis within a year of diagnosis. She remains to date without any major infectious, central nervous system (CNS) or autoimmune complications commonly seen in SIOD.

The majority of paediatric patients with NS respond to steroid treatment. A subset of children might however have clinical and biochemical features or a family history predictive of a steroid resistant NS. Recent advances in molecular genetics identified underlying genetic cause in a large proportion of patients with SRNS.

These children might benefit from an early referral to a tertiary centre in order to accelerate the diagnosis, potentially avoid renal biopsy and limit the exposure to steroids, which won't improve their condition. Rather they might increase their already elevated risk of infection and other complications.

\section{GP62 LIGNOCAINE TOXICITY: A CASE REPORT OF ADVERSE EFFECT OF LOCAL ANAESTHESIA IN COMMUNITY SETTING}

Nurrasyidah Abdul Halim*, Irina Chistol, Zachary Tan, Hafiz Shafiq Butt. St. Luke's General Hospital Carlow/Kilkenny, KILKENNY, Ireland

\subsection{6/archdischild-2019-epa.128}

Introduction Lignocaine is an amide-type local anaesthetic and a class Ib antidysrhythmic agent, available since 1948. Systemic exposure to large amounts leads to adverse effects on the cardiovascular and central nervous systems (CNS). Local anaesthetics are extremely useful for providing anaesthesia and analgesia for paediatric population of all ages. Our aim is to raise awareness among the clinicians regarding the safety of administration of local anaesthetics.

Case presentation 11 weeks old baby boy brought into the Paediatric Assessment Unit (PAU) by ambulance following an episode of respiratory depression and generalised tonic conic seizures in General Practice (GP) surgery. The patient had undergone a circumcision under local anaesthetia (brand name Xylocaine 1\%) shortly prior to the event. He required basic life support to maintain airway and breathing prior to ambulance arrival. Two doses on intranasal midazolam were administered by the paramedics to abort the seizures. A Guedel airway was inserted for airway protection and 100\% oxygen was supplied through the non-rebreather mask. 
On arrival, he had GCS of 6/15, pupils were pin point, capillary refill time (CRT) was 3-4seconds, tachycardic and hypothermic with temperature of $34.5 \mathrm{C}$. The rest of physical examinations were normal. No obvious haemorrhage on the circumcision site. He was given $20 \mathrm{mls} / \mathrm{kg}$ of normal saline and started on intravenous Cefotaxime. Temperature was stabilised with thermal mattress and continuous maintenance intravenous fluid and CRT had improved to 2 seconds. Lignocaine toxicity was suspected and he was given Intralipid $20 \%$ as per protocol. His conscious level improved momentarily following Intralipid administration but became impaired again. $\mathrm{He}$ was intubated and ventilated and was transferred to tertiary Paediatric Intensive Care Unit (PICU) for further management. He made a full recovery and was discharged home.

Discussion Overall safety record of local anaesthesia in paediatric population has been good provided administered within safe guidelines. Patients at the extremes of age and women who are pregnant more susceptible to local anaesthetic toxicity. Rates of severe systemic toxicity (seizures with or without cardiac arrest) occur up to 1:2000 for peripheral nerve blocks, depending on the type of block (Torp KD \& Simon LV, 2018). A retrospective study by Fotaine et al (1994), 1358 circumcised male infants revealed that 1022 had a dorsal penile nerve block (DPNB) as the anaesthetic technique and there were no reports of local anaesthetic toxicity in these cases. Despite the apparent safety of lignocaine, extra precautions should be applied in administering the medication in paediatric population. This case could be an example that despite the safety profile of lignocaine, severe toxicity still could occur.

\section{REFERENCES}

1. Fotaine P, Dittberner D, Scheltema KE. (1994) The safety of dorsal penile nerve block for neonatal circumcision. Journal of Family Practice.

2. Torp KD, Simon LV. (Updated 2018) Lidocaine Toxicity, Treasure Island (FL): StatPearls Publishing; 2018 Jan.

\section{GP63 PERIMENSTRUAL RHINITIS - A CASE REPORT}

K Flinn*, B Linnane. Department of Paediatrics, University Hospital Limerick, Limerick, Ireland

\subsection{6/archdischild-2019-epa.129}

Background and aims Rhinits is defined as the presence of one or more of the following symptoms: sneezing, anterior or posterior rhinorrhea, nasal congestion or nasal itchiness. The most common causes of rhinitis are allergic, nonallergic and mixed, however there are a number of others including hormonal rhinitis seen in pregnancy. There is also an identified link between menstruation and worsening of asthma symptoms, often referred to as perimenstrual asthma.

Our aim is to report the case of a teenage girl who presented with symptoms and signs of perimenstrual rhinitis.

Methods We describe the clinical presentation, management and outcome to date of a teenage girl with rhinitis associated with menstruation.

Results A fourteen year old girl was referred by her General Practitioner to the general paediatric outpatients with a presenting complaint of excessive clear rhinorrhea occurring with menstruation. The patient described the production of copious amounts of clear nasal discharge for approximately six days, starting three days prior to and finishing on day three of her period. The mucous production was so significant that it regularly resulted in mucous vomiting during this time. Her symptoms were ongoing since menarche at age eleven. She denied any other respiratory symptoms and did not complain of rhinorrhea at any other time. She did not have a history of asthma, eczema or hayfever. Prior to her appointment she had trialled an oral antihistamine at night from which she noted some improvement. She was an otherwise well teenager with no significant birth, past medical or family history. She had baseline bloods done which included FBC, UEC, LFT, CRP, Total IgE and IgE specific to dust mite, dander, grass and trees. Her Total IgE was slightly raised at 244.7 but all other investigations were within normal limits. Following her appointment she was commenced on the oral contraceptive pill. She was reviewed in clinic six months later and she noted a significant improvement in her symptoms since commencing the OCP. She no longer has any significant mucous production during her period.

Conclusion Rhinitis secondary to pregnancy has been described well in the literature, however there are only a handful of cases which describe menstrual related rhinitis. This case outlines a clear history with resolution of symptoms following regulation of the menstrual cycle with the OCP.

\section{GP64 POTTS PUFFY TUMORS, INFECTION, NOT TUMOR AND CAN KILL}

Zachary Tan*, Irina Chistol, Manana Pienaar, Victor Morris. St Luke's Hospital, Kilkenny, Ireland

\subsection{6/archdischild-2019-epa.130}

Introduction Potts Puffy tumor is defined as scalp swelling due to sub periosteal abscess with osteomyelitis of the frontal bone secondary to bacterial sinusitis. Sir Percival Pott first described the phenomenon in 1775 and since the advent of modern antibiotic therapy; this diagnosis is becoming a rare diagnosis. Case Presentation A 8 years old child without previous history of surgery or trauma, including no recent history of dental procedure, and no significant past medical history presented to the emergency department with swelling of her forehead and scalp, with headache for one day and being unwell for two days. On physical examination she had a soft boggy swelling in the forehead with extension towards the parietal bone. She had pyrexia, BP $136 / 91 \mathrm{mmHg}$, heart rate of 121 beats per minute. Her blood investigations revealed CRP - 141, WBC - 16.3, Hb - 11.4, neutrophils 10.73. U\&E, LFTs were normal and blood culture was negative. She had CT brain which showed extensive severe pan-sinusitis with associated abscess formation in the soft tissues underlying the frontal bone and intracranial abscess deep to right frontal bone. She was commenced on IV Co-amoxiclav and Flucloxacillin. She was transferred to the paediatric ENT team for drainage and wash out. The drainage and washout sample grew coagulase negative. She was commenced on ceftriaxone iv for 4 weeks, oral metronidazole $240 \mathrm{mg}$ po for six week and Fluticasone nasal spray for two weeks. She had full recovery with no further complications.

Discussion Potts puffy tumour can occur as a sequel of acute or chronic sinusitis. CT and MRI are important for diagnosis and monitoring. Prompt involvement of ENT and Neurosurgical team is required. Samples should be collected from drainage of pus for culture sensitivity as well as for 16s Ribosomal PCR. Antibiotics regimens usually employed are IV Ceftriaxone and Metronidazole both because of a good CNS penetration and Metronidazole being effective for all gram-negative anaerobes as well. 